\title{
BP-BEST-OP-4
}

\section{Evaluating the feasibility and effectiveness of extremely low-pressure pneumoperitoneum for robotic-assisted cholecystectomy}

\author{
Tae Ho HONG*, Sung Eun PARK
}

Department of Hepato-Biliary and Pancreas Surgery, Seoul St. Mary's Hospital, Seoul, Korea

Introduction: Standard-pressure pneumoperitoneum (12 to $15 \mathrm{mmHg}$ ) has been used to create an adequate working space during the laparoscopic surgery, but it has adverse effects such as postoperative pain, especially in the shoulder. Attempts are being made to reduce the postoperative pain by keeping low-pressure insufflation (7 to $10 \mathrm{mmHg}$ ). With advances in operative technology, the robotic-assisted cholecystectomy using da Vinci Xi could provide a sufficient surgical field with the extremely low-pressure pneumoperitoneum $(4 \mathrm{mmHg}$ ) by the videoscope pushing the abdominal wall upward. This study aimed to compare the postoperative outcomes using standard- and extremely low-pressure pneumoperitoneum in patients undergoing laparoscopic or robotic-assisted cholecystectomy.

Methods: A retrospective study was designed to compare the postoperative outcomes of 53 extremely low-pressure robotic-assisted cholecystectomy (ELRC), 116 standard-pressure robotic-assisted cholecystectomy (SRC) and 105 standard-pressure laparoscopic cholecystectomy (SLC).

Results: The surgery took longer in robotic-assisted cholecystectomy than laparoscopic cholecystectomy $(p<0.001)$. Postoperative pain score was significantly less in ELRC group as compared to SRC or SLC group at 6, 12, and 24 hours $(p=0.004, p=0.005$, and $p$ $=0.009$, respectively). However, there was no significant difference between groups in the number of demands for analgesic. The incidence of shoulder pain was significantly lower in ELRC group $(p<0.001)$. The rate of postoperative complication and length of stay were no difference between groups.

Conclusions: The use of the ELRC is associated with a significantly lower postoperative pain and shoulder pain. This study also shows that extremely low-pressure technique is feasible without increasing postoperative complications. 